EPJ Web of Conferences 59, 17007 (2013)

DOI: $10.1051 /$ epjconf/20135917007

(C) Owned by the authors, published by EDP Sciences, 2013

\title{
Enhanced energy coupling by using structured nano-wire targets
}

\author{
H. Habara ${ }^{1, a}$, Y. Mishima ${ }^{1}$, N. Nakanii ${ }^{1}$, S. Honda ${ }^{1, b}$, M. Katayama ${ }^{1}$, \\ L. Gremillet ${ }^{2}$, L. Willingale ${ }^{3}$, A. Maksimchuk ${ }^{3}$, K. Krushelnick ${ }^{3}$ \\ and K.A. Tanaka ${ }^{1}$
}

1 Graduate School of Engineering, Osaka University, 2-1 Yamada-oka, Suita, Osaka, Japan

2 CEA, DAM, DIF, 91297 Arpajon, France

3 Center for Ultrafast Optical Science, Nuclear Engineering and Radiological Sciences, University of Michigan, Ann Arbor, Michigan 48109-2099, USA

\begin{abstract}
We have investigated the interaction of ultra intense laser light with a carbon nanotube (CNT) target. The experimental results show an increased electron acceleration and a very low laser reflection as compared to non-structured targets. In addition, interferograms show very weak plasma expansion in front of the CNT target whereas the flat target creates a considerable amount of preformed plasma. A 2-D PIC calculation indicates that high laser absorption is possible via a Brunel mechanism following the ponderomotive heating in the expanded plasma between nanotubes.
\end{abstract}

\section{INTRODUCTION}

After the recent successful fast ignition (FI) integrated experiment at ILE, Osaka University [1], ultra high-intensity laser systems with considerable energy have been built for demonstration of FI such as GEKKO-LFEX and Omega-EP. However, more energetic, $100 \mathrm{~kJ}$-class laser pulses seem to be required for achieving ignition according to current estimates of the laser-to-plasma energy coupling efficiency [1]. This poses considerable technological challenges due to the difficulty of developing large optics with high energy tolerance such as compressor gratings.

An alternative approach is to increase the coupling efficiency itself. In a previous study, we reported on an increase of the conversion efficiency from laser light to the fast electrons by using a nano-size foam target [2]. A very thin gold foam material with about $400 \mathrm{~nm}$ diameter pores was irradiated on the GEKKO-PW laser light. Such nano-structured targets enable a concentration of the electromagnetic field at the target edges and excite "plasmons" around the structure, resulting in an efficient energy transfer from the laser to the material. Several similar studies have shown that targets consisting of aligned nano-tube/wires also allow for an enhanced energy coupling [3, 4]. However, these experiments were performed with $30 \mathrm{fs}$ laser systems at lower intensities than ours. It is therefore important to investigate whether these promising results can be extended to the high-intensity picosecond laser-plasma regime.

\footnotetext{
${ }^{a}$ e-mail: habara@eei.eng.osaka-u.ac.jp,

${ }^{\mathrm{b}}$ Current institution: Univ. Hyogo, Japan
}

This is an Open Access article distributed under the terms of the Creative Commons Attribution License 2.0, which permits unrestricted use, distribution, and reproduction in any medium, provided the original work is properly cited. 

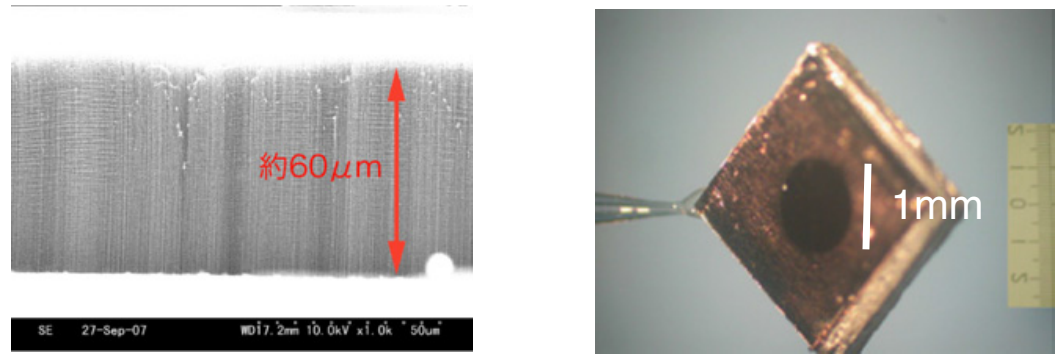

Figure 1. (Left) SEM image of CNT. (Right) CNT is aligned in circular shape on the copper plate.

\section{EXPERIMENTAL SETUP}

\subsection{Target}

Our target is made of aligned carbon nanotubes (CNTs) grown on a $10 \mu \mathrm{m}$ copper plate. Figure 1 shows a SEM image of the CNTs and of the whole target. The CNTs have a 60 to $100 \mu \mathrm{m}$ length with $15 \mathrm{~nm}$ diameter. Their areal density is around $80-100$ tubes $/ \mu \mathrm{m}^{2}$, resulting in nearly $1 / 100$ of density of solid graphite. As shown in the right picture of Figure 1, the CNTs are aligned circularly on the centre of the $\mathrm{Cu}$ foil. For comparison purposes, we used additional targets consisting of either a pure $\mathrm{Cu}$ foil or of a two-layer $75 \mu \mathrm{m}$-thick graphite/Cu foil.

\subsection{Experimental setup}

The experiments were performed on the GMII $60 \mathrm{TW}$ laser system at Osaka University and the T3 laser system at University of Michigan. Both systems comprise a Nd:glass CPA laser coupled to a Ti:Sapphire oscillator. The final pulse duration of both systems are similar $(400-500 \mathrm{fs})$. The $I \lambda^{2}$ of GMII and T 3 are $1 \times 10^{19}$ and $1 \times 10^{18} \mathrm{~W} \mu \mathrm{m}^{2} / \mathrm{cm}^{2}$, respectively. The contrasts for each laser are $10^{6}$ and $10^{9}$, respectively. In order to estimate the number of fast electrons, we measured the $\mathrm{Cu}-\mathrm{K} \alpha$ yield originating from the interaction of the fast electrons with $\mathrm{Cu}$ atoms via single photon counting method. Because the $\mathrm{Cu}$ foil is very thin, we expect that the electron energies inferred from the measurement ranged from several tens of $\mathrm{keV}$ to a few $\mathrm{MeV}$. An absolutely calibrated magnet electron spectrometer was used to diagnose the electron energy spectrum on the laser axis up to $300 \mathrm{MeV}$. In addition, the laser reflectivity was measured with a 7-channel fiber array set $28 \mathrm{~cm}$ away from the target, and covering $\pm 30^{\circ}$ in every $10^{\circ}$ viewing angles from the specular direction. Finally, an interferometric technique allowed us to monitor the plasma expansion 10 ps after the short-pulse irradiation.

\section{EXPERIMENTAL RESULTS}

\subsection{Fast electron creation}

Figures 2(a) shows typical electron spectra for CNT, graphite, and $\mathrm{Cu}$ targets. Although the number of fast electrons changes from shot to shot, their energy slope hardly varies as shown in Fig. 2(a): $\mathrm{T}_{\mathrm{h}}=$ $2.2( \pm 0.3) \mathrm{MeV}$ for $\mathrm{Cu}$ and $\mathrm{CNT}$, and 2.3 $( \pm 0.3) \mathrm{MeV}$ for graphite, respectively. These temperatures are two or three times higher than the usual ponderomotive estimate, which may imply the existence of a significant preformed plasma in front of the target. Fig. 2(b) plots the $\mathrm{Cu}-\mathrm{K} \alpha$ yield as a function of laser intensity. The yields associated to the CNT and graphite targets significantly differ at low intensities. At high intensities, the average $\mathrm{Cu}-\mathrm{K} \alpha$ yield from $\mathrm{CNT}$ target is found to be 2.3 times larger than that from graphite, and exceeds by $30 \%$ the yield from the $\mathrm{Cu}$ foil (the maximum error is $10 \%$ ) as observed in the previous literature [5,6]. The reduced difference in $\mathrm{K} \alpha$ yield at high intensities may 

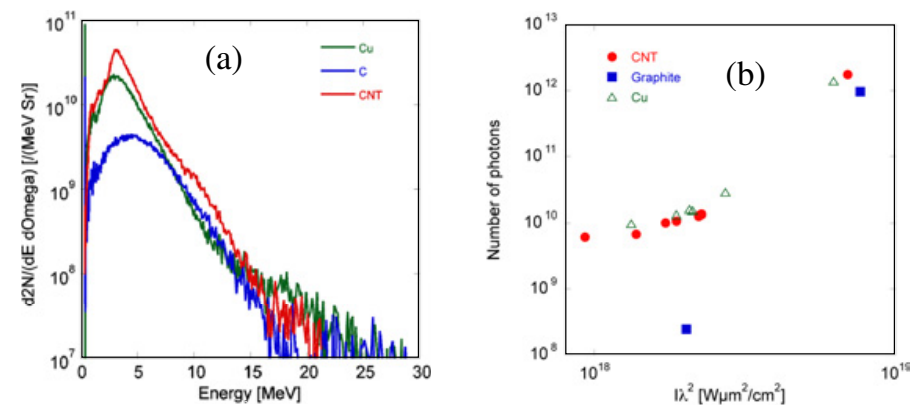

Figure 2. (a) Electron spectra at the highest laser intensity. (b) $\mathrm{Cu} \mathrm{K} \alpha$ intensity as a function of laser intensity from CNT (circles), graphite (squares) and copper (diamonds).
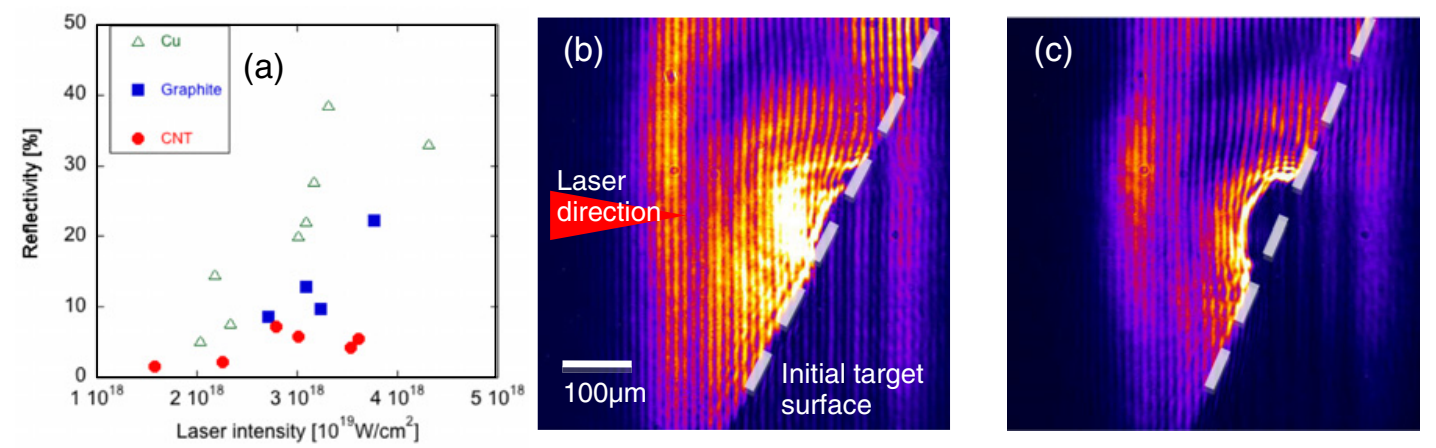

Figure 3. (a) Reflectivity of the laser light. (b) and (c) are interferometer images from CNT and graphite, respectively. Doted line represents the initial target surface.

stem from the expansion of the uniformization of the CNT plasmas during the interaction, resulting in plasma profiles in front of the $\mathrm{Cu}$ substrate similar to those obtained from non-structured targets. At any rate, the enhanced fast electron generation allowed by the CNT target may prove useful for FI-related applications.

\subsection{Reflection and absorption}

Figure 3(a) shows the laser reflectivity in the specular direction through the two-dimensional fitting deduced from the 7-fiber channel intensities. Our results pinpoint a nearly constant, very low reflectivity $(\sim 5 \%)$ in the case of CNT targets, consistently with previous experiments [7]. By contrast, the reflectivity associated to graphite and copper targets increases with the laser intensity. However, compared to Fig. 2, there is no clear relation between the laser absorption and fast electron generation. Understanding the dependence of the total energy balance on the target parameters requires further investigation. On the other hand, Fig. 3(b) and (c) display the interferometric images of the CNT and graphite targets for a laser intensity of about $8 \times 10^{18} \mathrm{~W} / \mathrm{cm}^{2}$. The $2 \omega$ probe light with 1.5 ps pulse duration is irradiated exactly perpendicular to the target surface. The images are recorded $10 \mathrm{ps}$ after the laser-target interaction. The laser pulse propagates rightward and the dashed line plots the initial target surface. The opaque area around the interaction spot corresponds to plasma densities higher than $6 \times 10^{19} \mathrm{~cm}^{-3}$. Note that the plasma expansion from the $\mathrm{Cu}$ target is similar to that observed from the graphite target, which agrees with the similar reflectivity signals measured at high intensities. By contrast, the CNT target exhibits a very limited plasma expansion in front of the target, consistently 

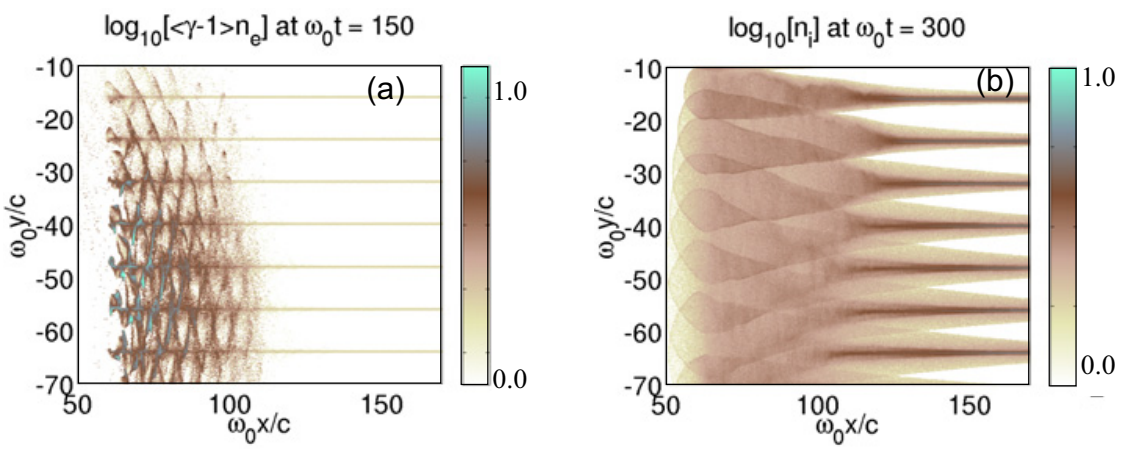

Figure 4. (a) Electron energy density distribution at $150 \omega_{0}$ t. (b) Ion density at $300 \omega_{0} \mathrm{t}$.

with the nearly constant reflectivity measured at high laser intensities. From the analysis of fringe shift, the tenuous plasma in front of CNT target expands nearly $100 \mu \mathrm{m}$, which is about half of that of the graphite target.

\section{PIC MODELING}

In order to understand the interaction of ultra intense laser light with CNT, a two-dimensional PIC simulation has been performed. The system size comprises $5000 \times 20000$ cells corresponding to $300 \times 400\left(\mathrm{c} / \omega_{0}\right)^{2}$ (where $\omega_{0}$ is the laser frequency). 300 macro-particles are used per cell. The maximum laser intensity is $4 \times 10^{21} \mathrm{Wcm}^{-2}$ with a $30 \mathrm{fs}$ pulse duration. The plasma consists of $\mathrm{C}^{6+}$ nanotubes with density of $230 n_{c}$ (where $n_{c}$ denotes the critical density) A density of one $\mathrm{CNT} / \mu \mathrm{m}$ is chosen in order to understand the absorption mechanism clearly and qualitatively.

Figure 4(a) shows the electron energy density at $t=150 \omega_{0}^{-1}$. The laser light propagates from the left to the right. The fast electrons are initially generated via a Brunel-like mechanism within the interference pattern formed by reflected/transmitted light around the nanotubes. A ponderomotive-like heating mechanism takes over when a few-critical plasma fills the vacuum gaps between nanotubes. The average electron temperature increases to $10 \mathrm{MeV}$. These absorption processes occur in each nanotube, leading to efficient laser absorption. On the other hand, Fig. 4(b) indicates the ion density distribution at $\mathrm{t}=$ $300 \omega_{0}^{-1}$. The strongly relativistic electron heating leads to the ions being accelerated preferentially in the radial direction via Coulomb explosion, in qualitative agreement with the experimental observation of a weak longitudinal ion expansion [Fig. 3(b)].

\section{SUMMARY}

We have investigated the interaction of ultra-intense laser light with CNT targets. Our results show efficient electron acceleration and very low laser reflection as compared to non-structured targets. In contrast to uniform targets whose front surfaces rapidly expand during and after the interaction, the CNT targets give rise to limited longitudinal plasma expansion. A PIC calculation reveals that the fast electron generation originates from successive Brunel-like and ponderomotive-like processes, as the CNT interspace becomes increasingly filled by radially expanding plasma. Our results suggest that strong laser absorption can be obtained using ultra-intense, relatively long-duration pulses by adjusting the nanotube parameters such as their length or density. With respect to FI studies, our results could motivate novel target designs consisting of attaching CNT on the inner tip of a cone-guided target. Higher conversion efficiencies along with limited preplasma formation could then be achieved. 
IFSA 2011

\section{References}

[1] R. Kodama et al., Nature (London) 418, 933 (2002)

[2] A.L. Lei et al., Phys. Rev. Lett. 96, 255006 (2006)

[3] T. Nishikawa et al., Appl. Phys. B 73, 185 (2001)

[4] R. Rajeev et al., Phys. Rev. Lett. 90, 115002 (2003)

[5] Y. Ji et al., Appl. Phys. Lett., 96, 041504 (2010)

[6] Z. Zhao, Phys. Plasmas 17, 123108 (2010)

[7] Z.P. Yang et al., Nano Lett. 8, 446 (2008) 